\title{
Perineural dexmedetomidine added to ropivacaine for ultrasound-guided erector spinae plane block prolongs analgesia duration and reduces postoperative opioid consumption after thoracotomy: a prospective, randomized study
}

\section{Qiang Wang}

National Cancer Center/National Clinical Research Center for Cancer/Cancer Hospital, Chinese Academy of Medical Sciences and Peking Union Medical College

Ruoshan Liu

National Cancer Center/National Clinical Research Center for Cancer/Cancer Hospital, Chinese Academy of Medical Sciences and Peking Union Medical College

Shijing Wei

National Cancer Center/National Clinical Research Center for Cancer/Cancer Hospital, Chinese Academy of Medical Sciences and Peking Union Medical College

\section{Guohua Zhang}

National Cancer Center/National Clinical Research Center for Cancer/Cancer Hospital, Chinese Academy of Medical Sciences and Peking Union Medical College

\section{Cheng Ni}

National Cancer Center/National Clinical Research Center for Cancer/Cancer Hospital, Chinese Academy of Medical Sciences and Peking Union Medical College

Li Sun

National Cancer Center/National Clinical Research Center for Cancer/Cancer Hospital, Chinese Academy of Medical Sciences and Peking Union Medical College

Hui Zheng ( $\square$ zhenghui_zlyy@163.com)

National Cancer Center/National Clinical Research Center for Cancer/Cancer Hospital, Chinese Academy of Medical Sciences and Peking Union Medical College https://orcid.org/0000-0002-2657-5377

\section{Research article}

Keywords: dexmedetomidine, open thoracic surgery, ultrasound-guided erector spinae plane block, pain, postoperative

Posted Date: December 21st, 2020 
DOl: https://doi.org/10.21203/rs.3.rs-130941/v1

License: (c) (1) This work is licensed under a Creative Commons Attribution 4.0 International License. Read Full License 


\section{Abstract}

Background: Single-injection erector spinae plane block (ESPB) has been reported to be successfully used for analgesia after open thoracotomy. However, the duration of analgesia is not long enough. Adding dexmedetomidine to local anesthetics is frequently used to prolong the duration of single-injection regional nerve block. This randomized study was designed to assess whether adding dexmedetomidine to ropivacaine for ESPB could effectively prolong the duration of analgesia and reduce opioid consumption after open thoracotomy.

Methods: Sixty patients with esophageal cancer were randomized to receive ESPB using $28 \mathrm{~mL}$ of $0.5 \%$ ropivacaine, with $2 \mathrm{~mL}$ of normal saline (group R) or $0.5 \mu \mathrm{g} / \mathrm{kg}$ dexmedetomidine in $2 \mathrm{~mL}$ (group RD) administered perineurally. ESPB was performed at the $5^{\text {th }}$ thoracic level under ultrasound guidance. The primary outcome was the duration of analgesia. The secondary outcomes were total postoperative sufentanil consumption, numerical rating scale pain scores, Ramsay sedation scale scores and adverse effects.

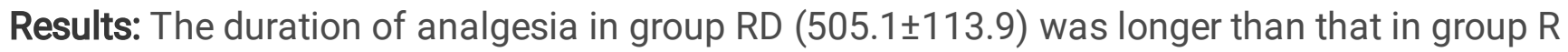
$(323.2 \pm 75.4)(p<0.001)$. The total postoperative sufentanil consumption was lower in group RD $(23.3 \pm 10.0)$ than in group $R(33.8 \pm 13.8)(p=0.001)$. There was no significant difference in the incidence of adverse effects between the two groups.

Conclusion: Adding perineural dexmedetomidine to ropivacaine for ESPB seems to be an attractive method for prolonging analgesia with almost no adverse effects in patients with esophageal cancer undergoing curative-intent thoracotomy.

Trial registration: ChiCTR1800016583. Registered 10 June 2018, http://www.chictr.org.cn.

\section{Background}

Open thoracotomy for the treatment of thoracic esophageal cancer is associated with intense acute postoperative pain. ${ }^{1}$ Single-injection erector spinae plane block (ESPB) has been reported to be successfully used for analgesia after thoracotomy. ${ }^{2}$ Although ESPB is effective for alleviating pain after thoracotomy, the duration of analgesia is not long enough. ${ }^{3}$ Because the moderate-severe acute postoperative pain associated with open thoracotomy frequently outlasts the duration of single-injection ESPB and there is muted enthusiasm regarding the use of continuous perineural catheters, the potential for prolonging the duration of analgesia after single-injection ESPB is especially important. ${ }^{4-6}$ Adding adjuvants to local anesthetics is frequently used to prolong the duration of single-injection regional nerve block. ${ }^{6-9}$ One of most frequently used adjuvants added to local anesthetics to prolong the duration of analgesia after single-injection nerve block is dexmedetomidine. ${ }^{6,8-10}$ As a meta-analysis and trial sequential analysis pointed out, in several kinds of peripheral nerve blocks, perineural dexmedetomidine can prolong the duration of analgesia. ${ }^{11}$ However, whether perineural dexmedetomidine prolongs the 
duration of single-injection ESPB and reduces postoperative opioid consumption after curative-intent open thoracotomy remains uncertain. Therefore, this prospective, randomized, double-blinded, controlled clinical study was performed to investigate whether adding dexmedetomidine to ropivacaine could prolong the duration of single-injection ESPB and reduce postoperative opioid consumption for open thoracotomy.

\section{Methods}

\section{Trial Design and participants}

This prospective, randomized, double-blinded, controlled clinical study was conducted in accordance with the Helsinki Declaration. The study was approved by the institutional ethics committee of National Cancer Center/National Clinical Research Center for Cancer/Cancer Hospital, Chinese Academy of Medical Sciences and Peking Union Medical College (18-079/1657). And this trial was registered on http://www.chictr.org.cn (ChiCTR1800016583) on June 10, 2018. The patients were enrolled on July 2, 2018. Written informed consent was obtained from every patient. A total of 113 patients with lower thoracic esophageal cancer, with an American Society of Anesthesiologists (ASA) physical status of I and II, aged 18 to 64 years old, and who were selected for open thoracic surgery (Sweet procedure) between July 2018 and December 2019 were recruited in this study. All patients were able to communicate well and understood how to evaluate their pain score at rest or during coughing. To eliminate potential confounders from the surgeons, all open thoracic surgeries were performed by only the same experienced surgical team ( $\mathrm{Li}$ Y, et al.). The major exclusion criteria were as follows: infections at the site of injection for ultrasound-guided ESPB; neuropathy; coagulation disorders; morbid obesity (body mass index more than $40 \mathrm{~kg} / \mathrm{m}^{2}$ ); allergy to ropivacaine or dexmedetomidine; adrenoreceptor agonist or antagonist therapy; greater than first-degree heart block; bradycardia (heart rate less than 50 beats per min); pregnancy; clinically significant cardiovascular, pulmonary, hepatic or renal diseases; psychiatric illnesses that would interfere with the assessment of pain scores; and those given a painkiller within one week before surgery. During the preoperative interview, patients were taught how to evaluate their pain score by the Numeric Rating Scale (NRS).

\section{Randomization And Blinding}

A computer-generated randomization list was used to randomize consented study participants on a 1:1 ratio to receive ultrasound-guided ESPB with the trial medication.

1. Group $\mathrm{R}$ (control): $28 \mathrm{~mL}$ of $0.5 \%$ ropivacaine $+2 \mathrm{~mL}$ of normal saline.

2. Group RD: $28 \mathrm{~mL}$ of $0.5 \%$ ropivacaine $+0.5 \mu \mathrm{g} / \mathrm{kg}$ in $2 \mathrm{~mL}$ of perineural dexmedetomidine.

The volume of the study drug was $30 \mathrm{~mL}$. None of the patients were administered intravenous dexmedetomidine perioperatively. The patient-controlled intravenous analgesia ( $\mathrm{PCIA}$ ) protocol was programmed with $200 \mu \mathrm{g}$ sufentanil diluted to $100 \mathrm{~mL}(1.5 \mathrm{~mL}$ bolus, lockout time interval of $10 \mathrm{~min}$ and 1 hour limit of $9 \mathrm{~mL}$ without any baseline infusion). The randomization table was kept in our hospital 
pharmacy. The study medication and PCIA solutions were prepared by a nurse who did not know the purpose of the study. The patients, surgeons and research staff who enrolled participants and collected study data were blinded to the group assignments.

\section{Interventions}

Before the study, every patient was trained on how to use the PCIA pump and instructed on using the NRS; with 0: no pain to 10: the worst imaginable pain).

Upon arrival in the operating room, routine monitoring for open thoracotomy, including five-lead electrocardiography, invasive blood pressure, heart rate, respiratory rate (RR) and pulse oxygen saturation $\left(\mathrm{SpO}_{2}\right)$, was applied.

Before general anesthesia induction, the patient was placed in a right lateral decubitus position, and a high-frequency linear ultrasound transducer (GE LOGIQe, Wauwatosa, Wisconsin) was placed in a longitudinal orientation $3 \mathrm{~cm}$ lateral to the T5 spinous process. Then, three muscles were identified superficial to the hyperechoic transverse process shadow as follows: trapezius, rhomboid major, and erector spinae. Under ultrasound guidance, an 8-cm 22-gauge block needle (Contiplex; B Braun, Melsungen, Germany) was inserted in-plane in a caudad-to-cephalad direction until the tip lay in the surface of the transverse process (Fig. 1A). Correct needle tip position was confirmed by visualizing linear fluid spread that separated the erector spinae muscle from the transverse process. Then, $30 \mathrm{ml}$ of the study drug was injected deep into the erector spinae muscle (Fig. 1B). The assessment of cutaneous sensory block by pinprick was initiated 30 min later by a blind observer. After confirmation and assessment of the sensory block to pinprick, general anesthesia was induced with $0.05 \mathrm{mg} / \mathrm{kg}$ midazolam, $1.5-2.5 \mathrm{mg} / \mathrm{kg}$ propofol, $0.2-0.4 \mu \mathrm{g} / \mathrm{kg}$ sufentanil and $0.15 \mathrm{mg} / \mathrm{kg}$ cisatracurium. General anesthesia was maintained with sevoflurane, sufentanil and cisatracurium.

The standardized anesthetic technique was used in the two groups. After anesthesia induction, a doublelumen endobronchial tube was placed under the guidance of fiberoptic bronchoscopy. Then, the patient was turned to a right lateral decubitus position from the supine position. The correct position of the double-lumen endobronchial tube was confirmed again by fiberoptic bronchoscopy. Before the skin incision, one-lung ventilation was initiated. The volume-controlled ventilation mode was adopted, with the airway pressure and lung compliance monitored. General anesthesia was maintained with $2.5 \%-3.0 \%$ sevoflurane (1.3-1.5 MAC). The end-tidal carbon dioxide partial pressure was maintained between 35 and $45 \mathrm{~mm} \mathrm{Hg}$ by mechanical ventilation.

After ipsilateral lung collapse, a posterolateral incision in the sixth intercostal space on the left side of the chest was made. At the conclusion of the surgical procedure, a chest drain was placed. At the end of the surgery, the intravenous analgesic pump was applied. PICA was administered when NRS $\geq 4$ or at the request of the patient.

\section{Outcomes}


The primary outcome was the duration of analgesia, which was defined as the time in minutes to the first request for postoperative analgesics. Total postoperative sufentanil consumption within the 48-hour period was also recorded. Before surgery (baseline) and at 3 hours, 6 hours, 12 hours, 24 hours, 36 hours and 48 hours after surgery, the patient's mean arterial pressure (MAP), heart rate (HR), NRS scores at rest and during coughing, and Ramsay sedation scale (RSS) (1: anxious; 2: cooperative and tranquil; 3 : responding to command; 4 : brisk response to stimuli; 5 : sluggish response to stimuli; and 6 : no response to stimuli) were all assessed.

Adverse effects such as hypotension (systolic blood pressure less than $90 \mathrm{mmHg}$ or more than a $20 \%$ decline from baseline), hypoxemia $\left(\mathrm{SpO}_{2}\right.$ less than $\left.90 \%\right)$, bradycardia (HR less than 50 beats per min), respiratory depression (RR less than 10 breaths per min lasting for more than $10 \mathrm{~min}$ ), pruritus, neurotoxicity, headache, nausea and vomiting were recorded after surgery. Hypotension was treated with $6 \mathrm{mg}$ ephedrine and $6 \mathrm{ml} / \mathrm{kg}$ normal saline; the same doses were repeated as required. Bradycardia was treated with intravenous boluses of $0.5 \mathrm{mg}$ atropine. Hypoxemia was treated with inhalation of pure oxygen through a face mask. Respiratory depression was treated with naloxone and oxygen until the respiratory rate was greater than 15 breaths per min.

\section{Statistical Analysis}

The primary outcome was the duration of analgesia by single-injection ESPB. We hypothesized that adding $0.5 \mu \mathrm{g} / \mathrm{kg}$ dexmedetomidine to $0.5 \%$ ropivacaine would prolong the duration of analgesia compared with only $0.5 \%$ ropivacaine after ESPB for open thoracotomy. In our preliminary study conducted in 10 adult patients ( 5 in each group), the mean duration of analgesia was $397 \pm 74$ min and $461 \pm 80$ min in group R and group RD, respectively. PASS version 11.0.7 (PASS, NCSS, LLC, USA) for Windows was used to calculate the sample size. Student's t-test was selected, and the group allocation ratio was equal. The hypothesized means of the analgesia duration were 397 and 461, and the standard deviations (SDs) were 74 and 80 , respectively. Then, we calculated that a sample of 24 patients would provide $80 \%$ power at a two-sided alpha level of 0.05 . Ultimately, we recruited 30 patients in each group for a total of 60 patients considering possible dropouts and incomplete follow-up.

The continuous variables are presented as the means \pm SDs or medians (25th to 75th percentiles), and categorical data are presented as numbers and percentages. Normality was tested by the KolmogorovSmirnov analysis. Student's t-test was used for analysis of the duration of analgesia, total postoperative sufentanil consumption, NRS score and RSS data. The Kaplan-Meier survival method was used for analysis of time-to-event outcomes, and the log-rank test was used to compare groups. For analysis of MAP and HR data, repeated measures ANOVA with Bonferroni correction was used. To analyze the incidence of adverse effects, Fisher's exact test was used. All data were processed by IBM SPSS Statistics 21.0 (IBM Inc., New York, NY). A 2-sided $p$ value less than 0.05 was considered to be statistically significant.

\section{Results}


A total of 113 patients were recruited to participate in this study from July 2018 to December 2019. In addition, 45 patients were ineligible because they did not meet the inclusion criteria or declined to participate. Eight patients were excluded from the trial because of non-adherence to the study protocol (5 patients were lost to follow-up; 1 patient underwent secondary emergency surgery in 48 hours; 1 patient refused to use PCIA after thoracotomy; and 1 patient developed atelectasis in 48 hours). Ultimately, 60 patients were randomized and completed the study protocol (group R: $n=30$; group RD: $n=30$ ). The Consolidated Standards of Reporting Trials flow diagram depicts participants progressing through the study phases (Fig. 2.).

Ultimately, a total of 60 patients were included in the analysis in this study. There were no significant differences in the patient characteristics between the groups (Table 1).

Table 1

Demographic data and surgical characteristics

\begin{tabular}{|llll|}
\hline & Group R & Group RD & p-Value \\
\hline Age (years) & $55.8 \pm 7.0$ & $57.9 \pm 6.0$ & 0.205 \\
\hline BMl $\left(\mathrm{kg} / \mathrm{m}^{2}\right)$ & $22.5 \pm 2.0$ & $22.8 \pm 2.2$ & 0.614 \\
\hline Sex $(\mathrm{F} / \mathrm{M})$ & $8 / 22$ & $9 / 21$ & 0.774 \\
\hline Duration of surgery $(\mathrm{min})$ & $141.8 \pm 32.9$ & $148.1 \pm 35.4$ & 0.473 \\
\hline Extubation time (min) & $21.6 \pm 5.8$ & $22.6 \pm 6.8$ & 0.553 \\
\hline
\end{tabular}

Data are expressed as the means \pm SDs for numbers. There were no significant differences between groups. BMI, body mass index; F, female; $M$, male; $R$, ropivacaine; $R D$, ropivacaine + dexmedetomidine.

The duration of analgesia was significantly longer in group RD than in group $R(p<0.001)$ (Table 2). Figure 3. represents the Kaplan-Meier survival analysis of the time to first analgesic request. The log-rank test revealed that the time to first analgesic request was prolonged in group RD compared with group $\mathrm{R}$ $(p<0.001)$.

Table 2

Duration of analgesia and total postoperative sufentanil consumption

\begin{tabular}{|llll|}
\hline Variable & Group R & Group RD & p-Value \\
\hline Duration of analgesia $(\mathrm{min})$ & $323.2 \pm 75.4$ & $505.1 \pm 113.9$ & $<0.001$ \\
\hline Total postoperative sufentanil consumption $(\mu \mathrm{g})$ & $33.8 \pm 13.8$ & $23.3 \pm 10.0$ & 0.001 \\
\hline $\begin{array}{l}\text { The duration of analgesia and total postoperative sufentanil consumption data are expressed as the } \\
\text { mean } \pm \text { SD. R, ropivacaine; RD, ropivacaine + dexmedetomidine. }\end{array}$ & \\
\hline
\end{tabular}


The total postoperative sufentanil consumption in group RD was significantly lower than that in group $\mathrm{R}$ $(p=0.001)($ Table 2).

At 12, 24, 36 and 48 hours postoperatively, the NRS score at rest in group R was higher than that in group $R D(p<0.05)(F i g .4 A)$. At 12, 24 and 36 hours postoperatively, the NRS score during coughing in group $R$ was higher than that in group RD $(p<0.05)$ (Fig. 4B).

The MAPs at different time points were significantly different in each group $(p<0.001)$ (Fig. 5.). Similarly, the discrepancies in HR at different time points were statistically significant $(p<0.01)$ (Fig. 6.). However, no significant differences were observed in the MAP or HR between group R and group RD $(p=0.250$ and $p=0.099$, respectively).

Table 3 shows the number of patients experiencing adverse effects. There were no significant differences in the incidences of bradycardia, hypotension, hypoxemia, respiratory depression, nausea, vomiting, pruritus, dizziness or neurotoxicity between group R and group RD. The RSS is shown in Fig. 7. At 12, 24, 36 , and 48 hours postoperatively, the RSS in group RD was higher than that in group $R(p<0.05)$.

Table 3

Incidence of adverse effects

\begin{tabular}{|llll|}
\hline Adverse effects & Group R & Group RD & p-Value \\
\hline Bradycardia & $0(0)$ & $0(0)$ & - \\
\hline Hypotension & $0(0)$ & $0(0)$ & - \\
\hline Hypoxemia & $0(0)$ & $0(0)$ & - \\
\hline Respiratory depression & $0(0)$ & $0(0)$ & - \\
\hline Nausea & $5(17)$ & $3(10)$ & 0.706 \\
\hline Vomiting & $3(10)$ & $1(3)$ & - \\
\hline Pruritus & $0(0)$ & $0(0)$ & - \\
\hline Dizziness & $3(10)$ & $2(7)$ & $0(0)$ \\
\hline Neurotoxicity & $0(0)$ & & -000 \\
\hline $\begin{array}{l}\text { The incidence of adverse effects data are expressed as numbers and percentages. R, ropivacaine; RD, } \\
\text { ropivacaine + dexmedetomidine }\end{array}$ & & \\
\hline
\end{tabular}

\section{Discussion}

In this study, we demonstrated that adding dexmedetomidine to ropivacaine could prolong the analgesia duration and reduce postoperative sufentanil consumption compared with ropivacaine alone in patients undergoing open thoracotomy without additional adverse effects. 
Previous studies have demonstrated that perineural dexmedetomidine in interfascial nerve block can prolong the analgesia time of ropivacaine. ${ }^{12,13}$ ESPB is a novel kind of interfascial nerve block that has gained popularity for providing effective analgesia for thoracic surgery. In the context of this knowledge, the following question can be raised: could dexmedetomidine prolong the analgesia time of ropivacaine in ESPB for open thoracotomy? Based on this study, we found that in open thoracotomy, which caused intense acute postoperative pain, adding $0.5 \mu \mathrm{g} / \mathrm{kg}$ dexmedetomidine to $0.5 \%$ ropivacaine significantly increased the analgesia time of ESPB. This interesting finding was in accordance with the work of Gao and colleagues, ${ }^{14}$ who observed that adding $1 \mu \mathrm{g} / \mathrm{kg}$ dexmedetomidine to $0.5 \%$ ropivacaine prolonged the analgesia time of ESPB for minimally invasive thoracoscopy. The exact mechanism of perineural dexmedetomidine for prolonging nerve block is still not fully understood, but it seems to be multifactorial. As previous studies have pointed out, the possible mechanisms by which perineural dexmedetomidine prolongs the duration of nerve block include vasoconstriction, inhibition of nociceptive afferent impulse transmission along myelinated C-fibers, inhibition of hyperpolarization-activated cation current, and the release of encephalin-like substances at peripheral sites. ${ }^{15,16}$ However, as an adjuvant to local anesthetics, dexmedetomidine might prolong the duration of regional nerve block through local or systemic effects. Unfortunately, similar to the flaw in the abovementioned studies (lack of an intravenous control group), this study could not determine whether the prolongation of analgesia was due to local or systemic effects.

In a previous study by $\mathrm{Xu}$ and colleagues, ${ }^{17}$ they found that adding $0.5 \mu \mathrm{g} / \mathrm{kg}$ dexmedetomidine to $0.25 \%$ ropivacaine for transversus abdominis plane block reduced the total amount of sufentanil in the first 24 hours after abdominal surgery. Similar to the results of their study, the total postoperative sufentanil consumption in this study was significantly lower in group RD than in group R in the first 48 hours after surgery, indicating that adding $0.5 \mu \mathrm{g} / \mathrm{kg}$ dexmedetomidine to $0.5 \%$ ropivacaine could provide a significant opioid-sparing effect after open thoracotomy. As Lee and colleagues found in their valuable study, patients with esophageal cancer undergoing curative-intent surgeries were particularly vulnerable to opioid abuse, which was probably associated with intense postsurgical pain after thoracotomy. ${ }^{18}$ Regional block for effectively managing postoperative pain while simultaneously decreasing opioid consumption was found to decrease subsequent opioid misuse or dependence. ${ }^{19,20}$ On the basis of the currently available evidence, we speculated that adding dexmedetomidine to ropivacaine for ESPB probably contributed to minimizing opioid use and misuse in patients with esophageal cancer undergoing curative-intent thoracotomy.

As demonstrated in this study, the RSS scores at 12, 24, 36, and 48 hours postoperatively were higher in group RD than in group R. Furthermore, the RSS score in group RD was lower than 3 points. These results indicated that perineural dexmedetomidine could provide a rational sedative effect in the first 48 hours after thoracotomy, including preventing patients from being restless, agitated or anxious. Despite perineural dexmedetomidine producing superior sedative effectiveness, no patients receiving dexmedetomidine experienced respiratory depression or desaturation in the first 48 hours after thoracotomy, which indicated that adding $0.5 \mu \mathrm{g} / \mathrm{kg}$ dexmedetomidine to ropivacaine for ESPB was safe. 
Although most available research has emphasized direct perineural mechanisms, ${ }^{16,21,22}$ in a previous interesting study, Hong and colleagues found that sedation with intravenous dexmedetomidine was able to prolong the analgesic effect of regional nerve block. ${ }^{23}$ However, the exact mechanism by which perineural dexmedetomidine prolongs the analgesia time of ESPB remains speculative.

As demonstrated in this study, adding $0.5 \mu \mathrm{g} / \mathrm{kg}$ dexmedetomidine to ESPB did not cause significant fluctuations in MAP or HR. Additionally, the incidence of other adverse effects, including nausea, vomiting and dizziness, was similar in the two groups. However, this result did not indicate that any dose of dexmedetomidine could be safely used for perineural injection. In a previous study by Esmaoglu and colleagues, ${ }^{24}$ they found that adding $100 \mu$ g dexmedetomidine to the local anesthetic caused obvious declines in systolic blood pressure, diastolic blood pressure and heart rate during the first 2 hours after surgery. In addition, a previous meta-analysis indicated that when the dose of dexmedetomidine for perineural injection was more than $50 \mu \mathrm{g}$, the possibility of intraoperative bradycardia significantly increased. ${ }^{25}$ These studies suggest that clinicians should select the dose of dexmedetomidine for perineural injection with caution.

There were several limitations in this study. First, there was no intravenous dexmedetomidine group in this study. Without intravenous control, it was difficult to ascertain whether the analgesic prolongation of dexmedetomidine was mediated by systemic or local perineural action. Second, the dose of dexmedetomidine selected in this study was based on our institution's anecdotal experience. Further investigation is still needed to determine the dose-ranging data. Third, considering that repeatedly assessing the cutaneous sensory block area after surgery can significantly disturb patients' rest, we used the time to first postoperative analgesic request instead of testing sensory block to ascertain the analgesia time of ESPB. However, the duration of postoperative pain relief might not be a reliable surrogate measure for the sensory blockade duration of ESPB. The former has been validated and extensively used to evaluate the effect of adjuncts on local anesthetic-based regional nerve block. ${ }^{26-30}$ Fourth, the patients might have fallen asleep when the investigators performed follow-up visits. This possibility might interfere with the measurements in this study. Fortunately, the randomized nature of this study probably balanced this situation. Fifth, the use of dexmedetomidine in this study was off-label. Although this drug has been widely used internationally and studied in many clinical trials, the United States Food and Drug Administration has not approved dexmedetomidine for perineural injection. Considering the inconclusive evidence on the safety of perineural dexmedetomidine, we would like to suggest that clinic practitioners gain approval from local regulatory health institutions for the off-label use of this drug.

\section{Conclusion}

In conclusion, our results suggest that in patients with esophageal cancer undergoing curative-intent open thoracotomy, adding $0.5 \mu \mathrm{g} / \mathrm{kg}$ dexmedetomidine to single-injection ESPB with $0.5 \%$ ropivacaine 
prolongs the duration of analgesia and reduces postoperative opioid consumption without producing evident adverse effects.

\section{Abbreviations}

ESPB, erector spinae plane block; NRS, Numeric Rating Scale; PCIA, patient-controlled intravenous analgesia; RR, respiratory rate; $\mathrm{SpO}_{2}$, pulse oxygen saturation; $\mathrm{MAP}$, mean arterial pressure; HR, heart rate; RSS, Ramsay sedation scale; SDs, standard deviations; BMI, body mass index; F, female; M, male; R, ropivacaine; RD, ropivacaine + dexmedetomidine;

\section{Declarations}

Ethics approval and consent to participate: This study was approved by the institutional ethics committee of the National Cancer Center/National Clinical Research Center for Cancer/Cancer Hospital, Chinese Academy of Medical Sciences and Peking Union Medical College (18-079/1657) and registered on Chictr.org.cn (ChiCTR1800016583). Written informed consent was obtained from every patient.

Consent to publish: The manuscript has been approved by all authors for publication and no conflict of interest exits in the submission of this manuscript.

Availability of data and materials: The data and materials can be obtained by contacting the corresponding author.

Competing Interests: The authors declare no conflicts of interests.

Funding: This study was funded by the Beijing Hope Run Special Fund of Cancer Foundation of China (grant LC2017A09), the Management Research Project Special Fund of Cancer Hospital Chinese Academy of Medical Sciences (grant LC2018D01) and Sanming Project of Medicine in Shenzhen (grant SZSM201812069), Cancer Pain Treatment and Perioperative Medical Team of professor Sun Li in Cancer Hospital, Chinese Academy of Medical Sciences. The funding had no role in the study design, data collection or analysis or in the decision to publish or prepare the manuscript.

Authors' contributions: QW contributed to the formal analysis, investigation, writing of the original draft and writing of the review \& editing; RS L contributed to the data curation and investigation; SJ W contributed to the data curation and investigation; $\mathrm{GH} Z$ contributed to the formal analysis, investigation and project administration; $\mathrm{CN}$ contributed to the investigation, methodology and project administration; LS contributed to the formal analysis and methodology; $\mathrm{HZ}$ contributed to the acquisition of funding, supervision and writing of the review \& editing. All authors have read and approved the final manuscript.

Acknowledgements: Not applicable.

\section{References}


1. Miyata K, Fukaya M, Itatsu K, et al. Muscle sparing thoracotomy for esophageal cancer: a comparison with posterolateral thoracotomy. Surg Today. 2016; 46: 807-14. doi: 10.1007/s00595-015-1240-1.

2. Fang $B$, Wang Z, Huang X. Ultrasound-guided preoperative single-dose erector spinae plane block provides comparable analgesia to thoracic paravertebral block following thoracotomy: a single center randomized controlled double-blind study. Ann Transl Med. 2019; 7:174. doi: 10.21037/atm.2019.03.53.

3. Tulgar S, Kapakli MS, Senturk O, et al. Evaluation of ultrasound-guided erector spinae plane block for postoperative analgesia in laparoscopic cholecystectomy: A prospective, randomized, controlled clinical trial. J Clin Anesth. 2018; 49: 101-6. doi: 10.1016/j.jclinane.2018.06.019.

4. Raft J, Chin KJ, Belanger ME, et al. Continuous erector spinae plane block for thoracotomy analgesia after epidural failure. J Clin Anesth. 2019; 54:132-3. doi: 10.1016/j.jclinane.2018.10.024.

5. Yu Y, Liu N, Zeng Q, et al. The efficacy of pregabalin for the management of acute and chronic postoperative pain in thoracotomy: a meta-analysis with trial sequential analysis of randomizedcontrolled trials. J Pain Res. 2018; 12: 159-70. doi: 10.2147/JPR.S183411.

6. Zhang P, Liu S, Zhu J, et al. Dexamethasone and dexmedetomidine as adjuvants to local anesthetic mixture in intercostal nerve block for thoracoscopic pneumonectomy: a prospective randomized study. Reg Anesth Pain Med. 2019 Aug 8. doi: 10.1136/rapm-2018-100221. doi: 10.1136/rapm-2018-100221.

7. Zhao XY, Zhang EF, Bai XL, et al. Ultrasound-guided continuous femoral nerve block with dexmedetomidine combined with low concentrations of ropivacaine for postoperative analgesia in elderly knee arthroplasty. Med Princ Pract. 2019; 28: 457-62. doi: 10.1159/000500261.

8. Hong B, Lim C, Kang H, et al. Thoracic paravertebral block with adjuvant dexmedetomidine in videoassisted thoracoscopic surgery: a randomized, double-blind study. J Clin Med. 2019; 8: doi: $10.3390 / \mathrm{jcm} 8030352$.

9. Li J, Yang JS, Dong BH, et al. The effect of dexmedetomidine added to preemptive ropivacaine infiltration on postoperative pain after lumbar fusion surgery: a randomized cntrolled t Spine (Phila Pa 1976). 2019; 44: 1333-8. doi: 10.1097/BRS.0000000000003096.

10. Thapa D, Ahuja V, Pandey K, et al. Evaluation of analgesic efficacy of dexmedetomidine as adjuvant with ropivacaine in ultrasound-guided adductor canal block in patients following anterior cruciate ligament reconstruction surgeries. Br J Pain. 2019; 13: 91-8. doi: 10.1177/2049463718796865.

11. Schnabel A, Reichl SU, Weibel S, et al. Efficacy and safety of dexmedetomidine in peripheral nerve blocks: a meta-analysis and trial sequential analysis. Eur J Anaesthesiol. 2018; 35: 745-58. doi: 10.1097/EJA.0000000000000870.

12. Chen Q, Liu X, Zhong X, et al. Addition of dexmedetomidine or fentanyl to ropivacaine for transversus abdominis plane block: evaluation of effect on postoperative pain and quality of recovery in 
gynecological surgery. J Pain Res. 2018; 11: 2897-903. doi: 10.2147/JPR.S178516.

13. Ding W, Li W, Zeng X, et al. Effect of adding dexmedetomidine to ropivacaine on ultrasound-guided dual transversus abdominis plane block after g J Gastrointest Surg. 2017; 21: 936-46. doi:

$10.1007 / \mathrm{s} 11605-017-3402-5$.

14. Gao Z, Xiao Y, Wang Q, et al. Comparison of dexmedetomidine and dexamethasone as adjuvant for ropivacaine in ultrasound-guided erector spinae plane block for video-assisted thoracoscopic lobectomy surgery: a randomized, double-blind, placebo-controlled trial. Ann Transl Med. 2019; 7: 668. doi: 10.21037/atm.2019.10.74.

15. Yabuki A, Higuchi $H$, Yoshitomi T, et al. Locally injected dexmedetomidine induces vasoconstriction via peripheral a-2A adrenoceptor subtype in guinea pigs. Reg Anesth Pain Med. 2014; 39: 133-6. doi: 10.1097/AAP.0000000000000048.

16. Brummett CM, Hong EK, Janda AM, et al. Perineural dexmedetomidine added to ropivacaine for sciatic nerve block in rats prolongs the duration of analgesia by blocking the hyperpolarization-activated cation current. Anesthesiology. 2011; 115: 836-43. doi: 10.1097/ALN.0b013e318221fcc9.

17. Xu L, Hu Z, Shen J, et al. Efficacy of US-guided transversus abdominis plane block and rectus sheath block with ropivacaine and dexmedetomidine in elderly high-risk patients. Minerva Anestesiol. 2018; 84: 18-24. doi: 10.23736/S0375-9393.17.11538-5.

18. Lee JS, Hu HM, Edelman AL, et al. New persistent opioid use among patients with cancer after curative-intent surgery. J Clin Oncol. 2017; 35: 4042-9. doi: 10.1200/JC0.2017.74.1363.

19. Trasolini NA, McKnight BM, Dorr LD. The opioid crisis and the orthopedic s J Arthroplasty. 2018; 33 : 3379-82.e1. doi: 10.1016/j.arth.2018.07.002.

20. Soffin EM, Lee BH, Kumar KK, et al. The prescription opioid crisis: role of the anaesthesiologist in reducing opioid use and misuse. Br J Anaesth. 2019; 122: e198-e208. doi: 10.1016/j.bja.2018.11.019.

21. Brummett CM, Padda AK, Amodeo FS, et al. Perineural dexmedetomidine added to ropivacaine causes a dose-dependent increase in the duration of thermal antinociception in sciatic nerve block in rat. Anesthesiology. 2009; 111: 1111-9. doi: 10.1097/ALN.0b013e3181bbcc26.

22. Brummett CM, Amodeo FS, Janda AM, et al. Perineural dexmedetomidine provides an increased duration of analgesia to a thermal stimulus when compared with a systemic control in a rat sciatic nerve block. Reg Anesth Pain Med. 2010; 35: 427-31. doi: 10.1097/AAP.0b013e3181 ef4cf0.

23. Hong $B$, Jung $C$, Jo $Y$, et al. Sedation with dexmedetomidine prolongs the analgesic duration of brachial plexus block: a randomised controlled trial. Anaesth Crit Care Pain Med. 2019; 38: 231-6. doi: 10.1016/j.accpm.2018.08.006. 
24. Esmaoglu A, Yegenoglu F, Akin A, et al. Dexmedetomidine added to levobupivacaine prolongs axillary brachial plexus block. Anesth Analg. 2010; 111: 1548-51. doi: 10.1213/ANE.0b013e3181fa3095.

25. Hussain N, Grzywacz VP, Ferreri CA, et al. Investigating the efficacy of dexmedetomidine as an adjuvant to local anesthesia in brachial plexus block: a systematic review and meta-analysis of 18 randomized controlled trials. Reg Anesth Pain Med. 2017; 42:184-96. doi:

10.1097/AAP.0000000000000564.

26. Abdallah FW, Johnson J, Chan V, et al. Intravenous dexamethasone and perineural dexamethasone similarly prolong the duration of analgesia after supraclavicular brachial plexus block: a randomized, triple-arm, double-blind, placebo-controlled trial. Reg Anesth Pain Med. 2015; 40: 125-32. doi: 10.1097/AAP.0000000000000210.

27. Parrington SJ, O'Donnell D, Chan VWS, et al. Dexamethasone added to mepivacaine prolongs the duration of analgesia after supraclavicular brachial plexus blockade. Reg Anesth Pain Med. 2010; 35: 422-6. doi: 10.1097/AAP.0b013e3181e85eb9.

28. Cummings KC 3rd, Napierkowski DE, Parra-Sanchez I, et al. Effect of dexamethasone on the duration of interscalene nerve blocks with ropivacaine or bupivacaine. Br J Anaesth. 2011; 107: 446-53. doi: 10.1093/bja/aer159.

29. Desmet $\mathrm{M}, \mathrm{Braems} \mathrm{H}$, Reynvoet $\mathrm{M}$, et al. V. and perineural dexamethasone are equivalent in increasing the analgesic duration of a single-shot interscalene block with ropivacaine for shoulder surgery: a prospective, randomized, placebo-controlled study. Br J Anaesth. 2013; 111: 445-52. doi: 10.1093/bja/aet109.

30. Rahangdale R, Kendall MC, McCarthy RJ, et al. The effects of perineural versus intravenous dexamethasone on sciatic nerve blockade outcomes: a randomized, double-blind, placebo-controlled study. Anesth Analg. 2014; 118:1113-9. doi: 10.1213/ANE.0000000000000137.

\section{Figures}




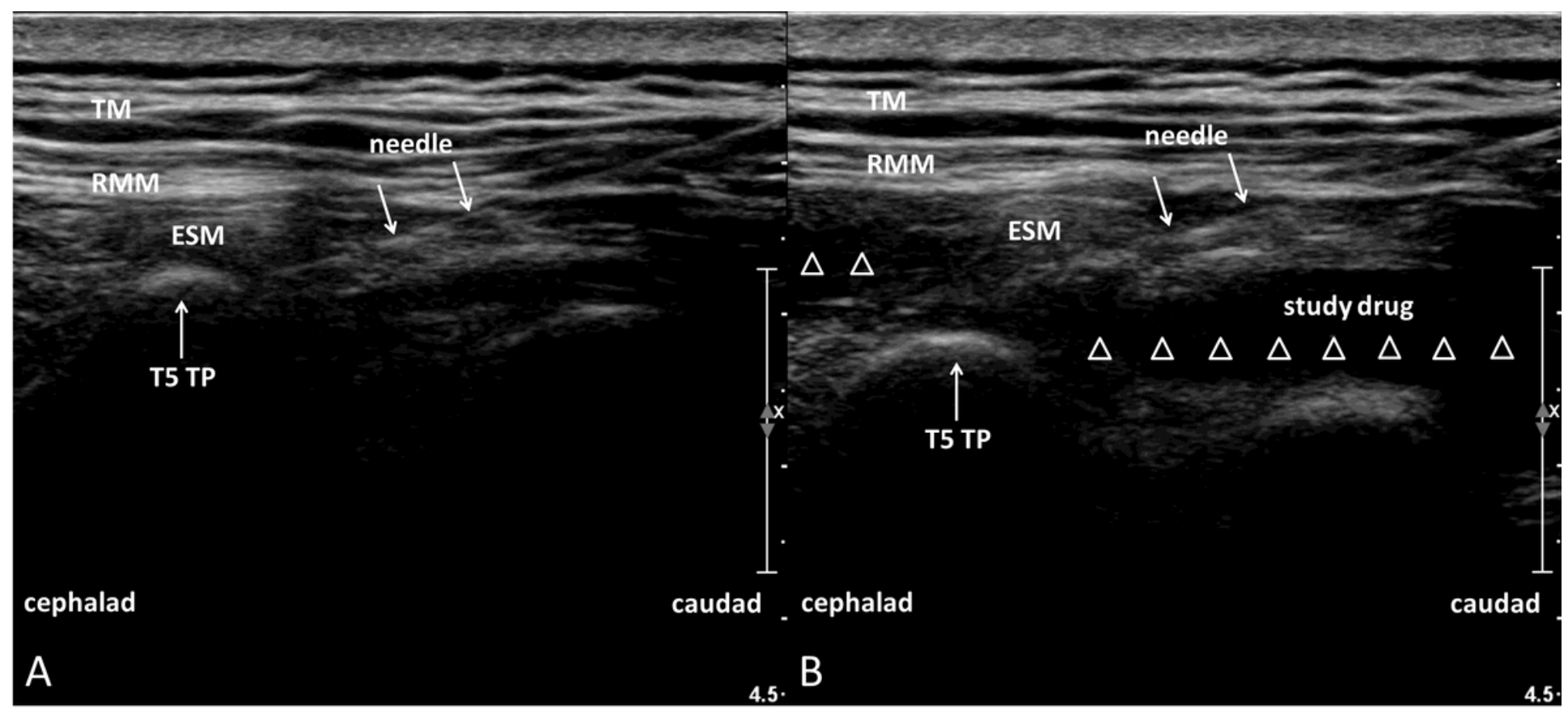

\section{Figure 1}

Ultrasound-guided ESPB performed deep to the ESM. (A) Ultrasound imaging before injecting study drug. (B) Injection of study drug into the interfascial plane deep to ESM produced a visible fluid spread (white hollow triangles) beneath the ESM. Abbreviations: ESPB, erector spinae plane block; TM, trapezius; RMM, rhomboid major; ESM, erector spinae muscle; T5, the fifth thoracic vertebra; TP, transverse process. 


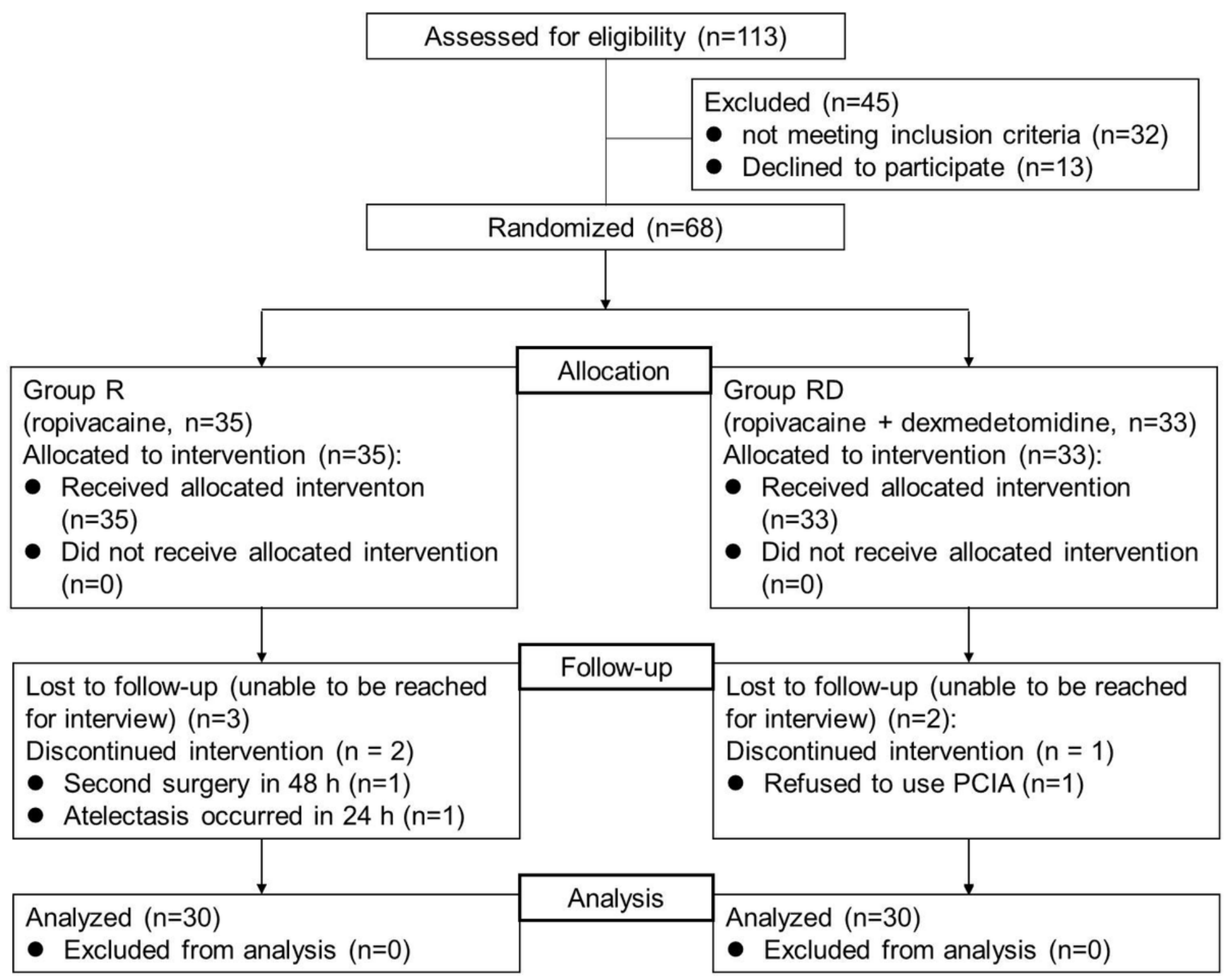

Figure 2

Patient enrollment and inclusion and exclusion process. Abbreviations: PCIA, patient-controlled intravenous analgesia. 


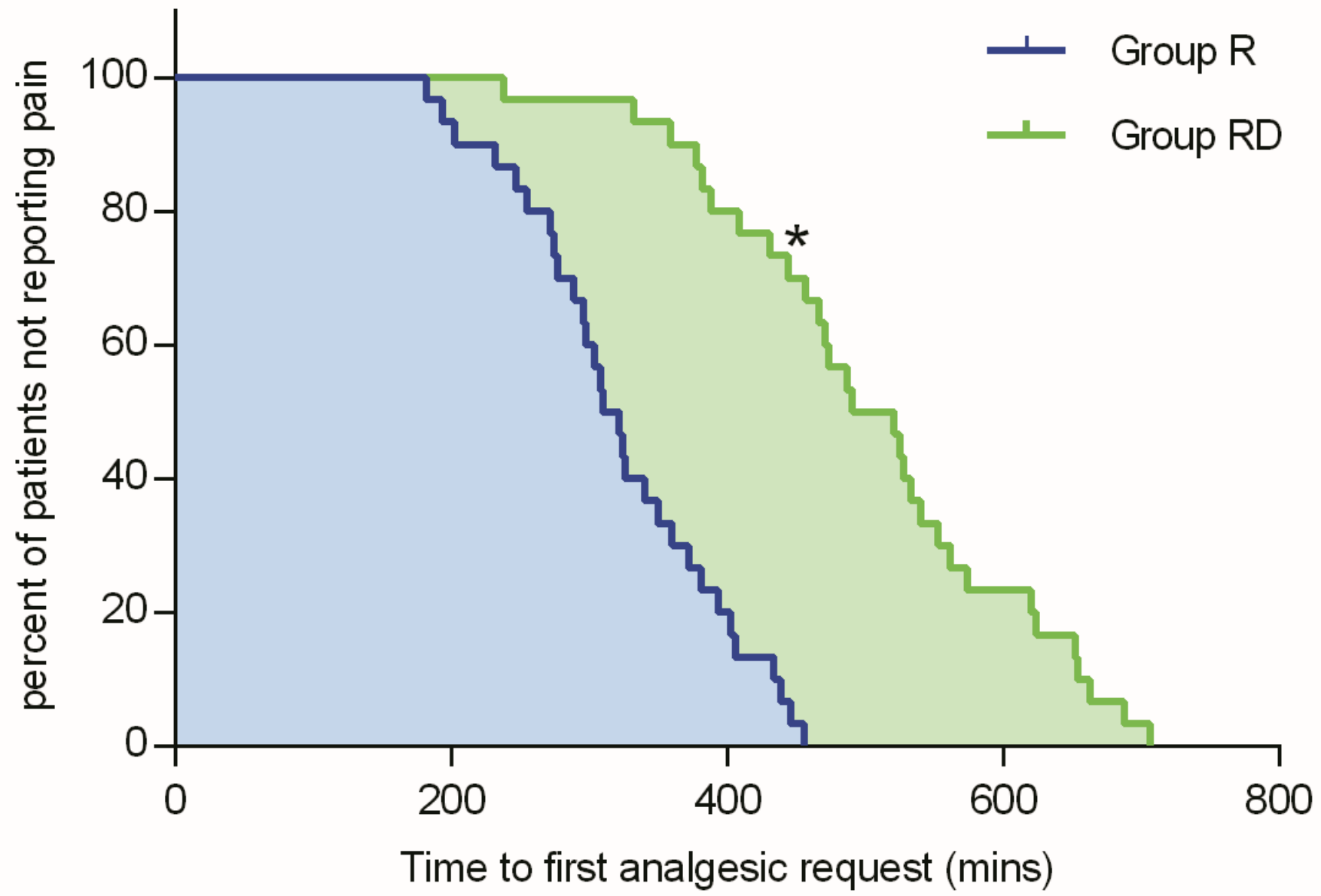

Figure 3

Kaplan-Meier survival plot representing the first analgesic request in the two groups. ${ }^{*} \mathrm{P}<0.001$ between group RD and group R. Abbreviations: R, ropivacaine; RD, ropivacaine + dexmedetomidine.

$\square$ group $\mathrm{R}$

group RD
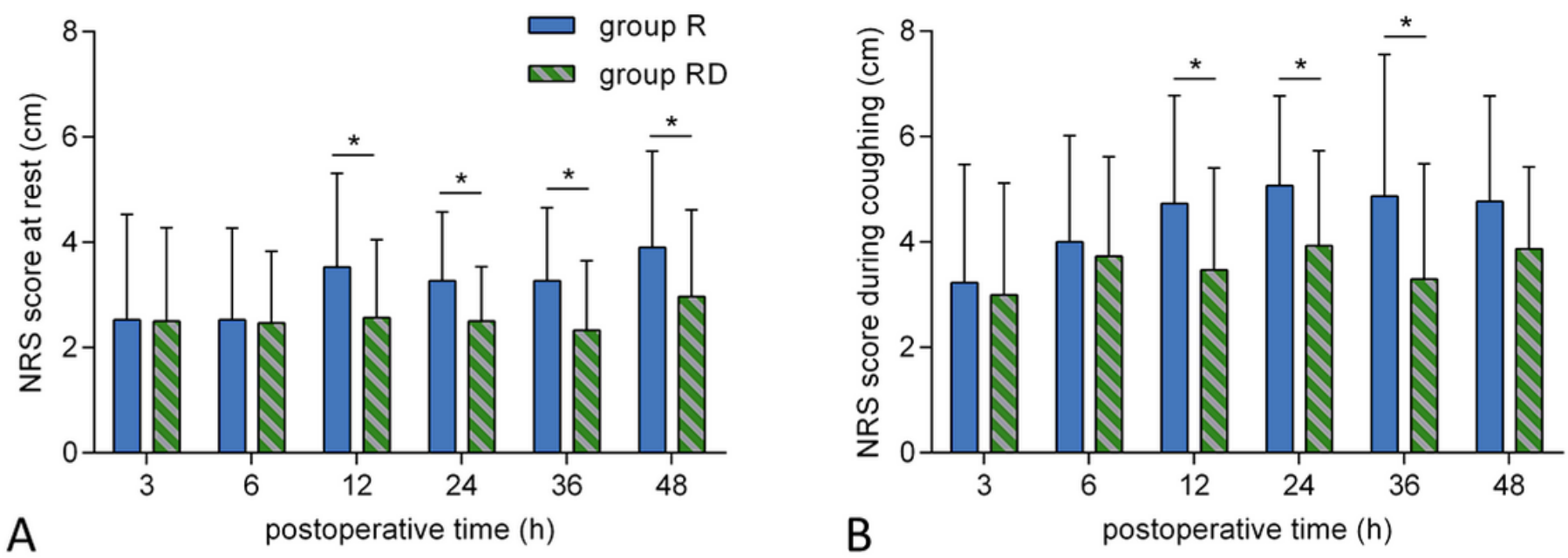
Figure 4

Postoperative pain severity NRS score at rest (A) and during coughing (B) at 3, 6, 12, 24, 36 and 48 hours postoperatively. Abbreviations: R, ropivacaine; RD, ropivacaine + dexmedetomidine; NRS, Numeric Rating Scale. ${ }^{*} \mathrm{P}<0.05$.

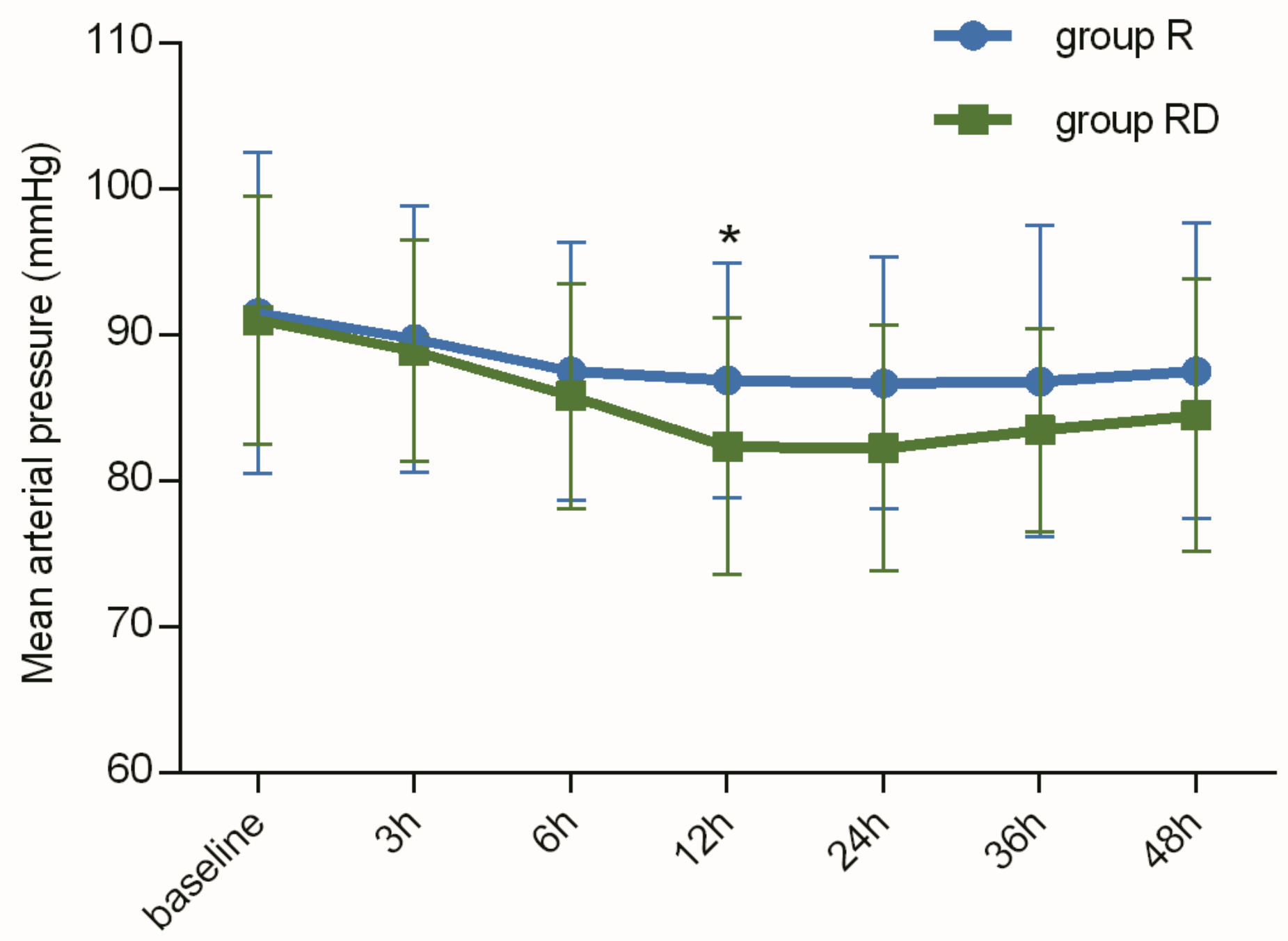

Time interval

\section{Figure 5}

Mean arterial pressure (MAP) changes at different times in each group. In both groups, the changes in MAP showed a significant difference in relation to time, with $* P<0.001$. 


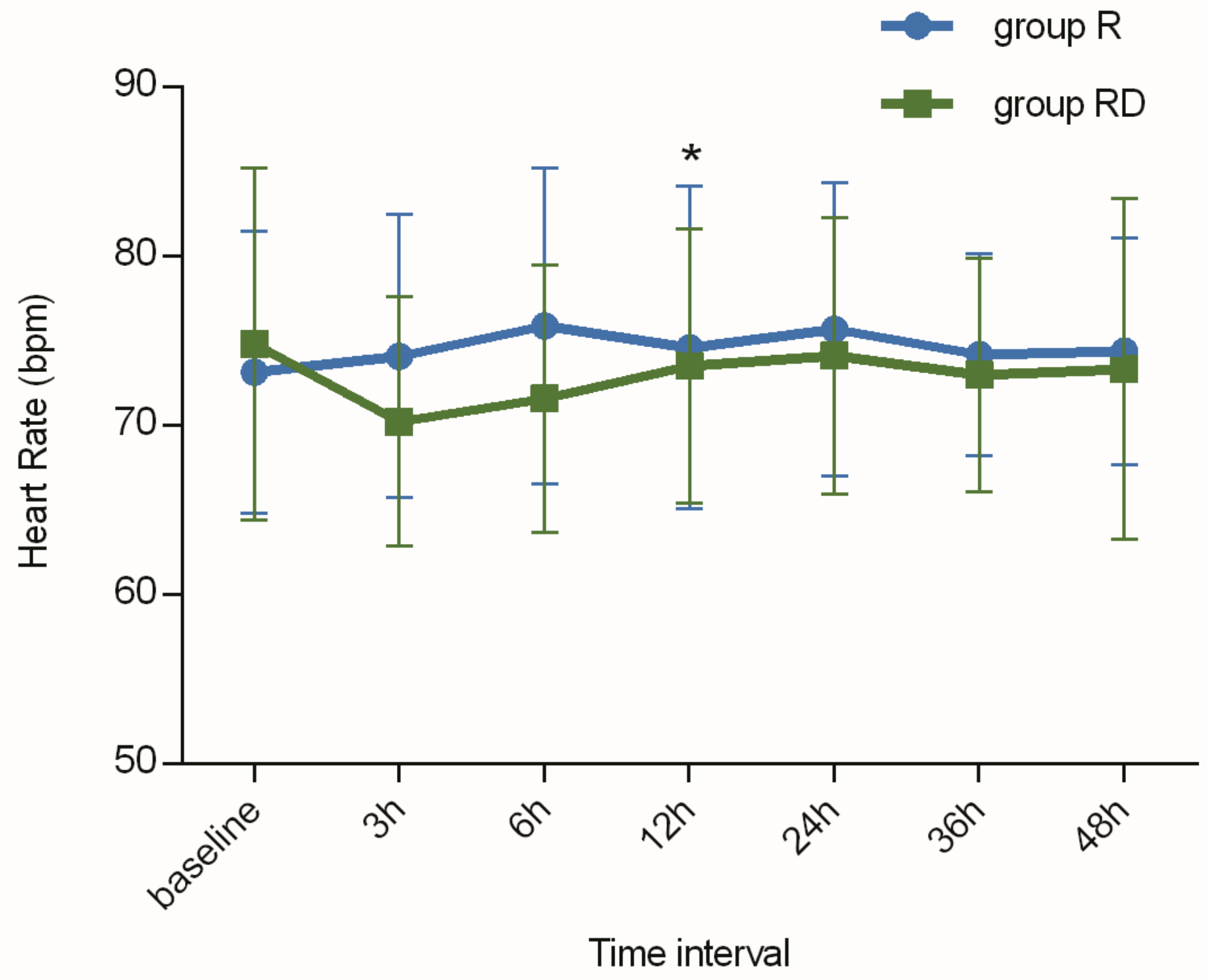

Figure 6

Heart rate changes at different times in each group. In both groups, the changes in heart rate showed a significant difference in relation to time with ${ }^{*} P<0.01$. Abbreviations: $R$, ropivacaine; $R D$, ropivacaine + dexmedetomidine. 


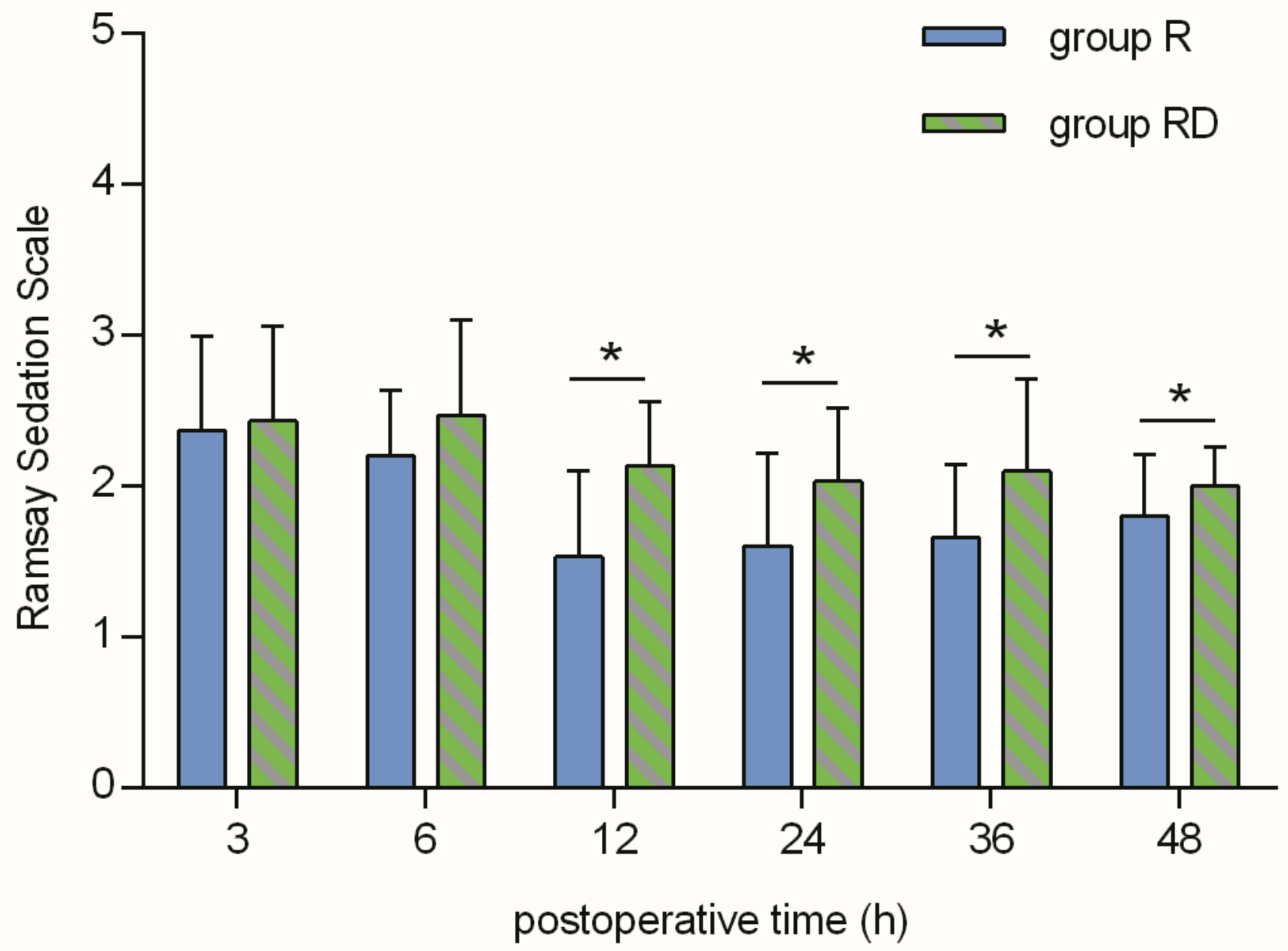

Figure 7

Ramsay Sedation Scale scores at 3, 6, 12, 24, 36 and 48 hours postoperatively. Abbreviations: R, ropivacaine; $\mathrm{RD}$, ropivacaine + dexmedetomidine. ${ }^{*} \mathrm{P}<0.05$.

\section{Supplementary Files}

This is a list of supplementary files associated with this preprint. Click to download.

- CONSORT2010Checklist.doc

- CONSORT2010FlowDiagram.doc 